\title{
Flood Prediction and Prevention through Wireless Sensor Networking (WSN): A Survey
}

\author{
Indira Priyadarshinee \\ Master of Technology \\ Dept. of Computer Sc \\ Centurion Inst. of Technology, \\ Odisha
}

\author{
Kabita Sahoo \\ Asst. Professor \\ Dept. of Computer Sc \\ Centurion Inst. of Technology, \\ Odisha
}

\author{
Chandrakant Mallick \\ Asst. Professor \\ Dept. of Computer Sc \\ Centurion Inst. of Technology, \\ Odisha
}

\begin{abstract}
Natural disaster is a usual phenomenon by which the living as well as nonliving entities belong to the environment is suffering regularly. Human being does not have the power to totally uproot the natural calamity but the only thing Human can do is, it can predict natural calamity \& take major steps to prevent it.

There are many technologies available to predict and prevent the natural calamity. In this paper we are employing Wireless Sensor Networking (WSN) technology for predicting \& preventing the flooding condition.

WSN is preferred due to its cost effectiveness, faster transfer of data $\&$ accurate computation of required parameter for flood prediction \& prevention. Another beauty of the WSN technology is that we could compute the required parameter by considering very few number of environmental parameter. In this paper a survey is done on flood prediction \& prevention through wireless sensor network.
\end{abstract}

\section{Keywords}

Wireless Sensor Networking (WSN), Flood prediction, Flood modeling, Sensor, Computational node.

\section{INTRODUCTION}

Flood is a kind of natural calamity. In an informal way, flood can be combination of flow \& odd means a typical odd flow of surplus water causes flood. The main cause of flood is the hydrological condition [15] of discharge of heavy rain fall and the enormous water. The other associated cause is metrological condition i.e. untimely cycle \& storm. The planning problem i.e. poor drainage, high siltation in river, breaching of the embankments, spilling of floodwaters over them also contributes to the flooding situation. The geographical condition i.e. flows of water from neighboring states, flow of river across the state \& the topographical condition up to some extent are the factor of flooding condition. In developed country like US, Japan etc the flooding problem is minimized significantly \& do not affect much due to availability of emergency system. But in developing country like India, Brazil etc it is suffering a lot during flood. Taking the state Odisha, as an instance the economic loss for the year 2013 is noticeable. In Odisha, Flood situation is a troublesome situation which occurs almost every year, to say regularly \& washes away the economy of Odisha. Most of the districts are affected by flood. In 2013 Odisha has faced a subsequent flood situation due to cyclone Phailin. From this perspective, the struggle with flooding for the poor and developing countries presents an urging issue that we cannot ignore simply while promising a solution that is globally applicable.
Flood detection \& prevention is a very expensive process in recent strategy .Current methods add to the difficulty with the need for expensive equipment, centralized and computationally difficult flood detection schemes.

The flood warning system used in the developed world are costlier and depends on the expert hydrologists who monitor real-time data 24 hours a day and run sophisticated computational models at a centralized location. These kinds of resources are unaffordable $\&$ impractical for poor counties as well as developing country.

Floods ends with the loss of numerous lives and leaves the flooded area with huge destruction of property every year, especially the rage of flood in the poor and developing countries is most noticeable, where people are the victim of the natural clemency.

A lot of techniques has been developed which could help us to minimize the damage through early disaster predictions. In deploying a prediction model in the rural areas, there are a number of severe limitations of resources like money, power and skilled man-power [1].

This motivates us to utilize the work of information communication technology \& sensor network to solve the networks to overcome several problems associated with developing and poor country for fighting with the flood. This could also help in a way to balance the minimal cost requirement and limited computational power with high reliability of both the system and computation.

In this paper we have described different research work on flood prediction and prevention through wireless sensor network (WSN). The previously worked paper on flood prediction using a WSN and other relative papers invigorate us the different ways to predict flood (flood forecast). These reference papers are providing the ideas how to minimize its destructive effects of flood on Human being to save the lives and property by using a the newly evolved technology called as Wireless Sensor Network. Especially in our proposed work WSN is taken into account for the flood prediction \& its effect minimization in an innovative way.

\section{LITERATURE REVIEWS}

A reliable computational model which could fight with the flood in developing and poor countries is our main concern. We are describing different research work that are deploying wireless sensor network (WSN).There are different models, the different energy efficiency models, different networking arrangement of wireless sensor networks. These motivate us in preparing a most efficient model for predicting and preventing flood. 


\subsection{Flood Alarming}

Seal et al.[1] presents a forecasting model designed using WSNs( Wireless Sensor Networks).This model helps to predict flood in rivers using simple and fast calculations to provide real-time results and save the lives of people who may be affected by the flood by ringing a alarm. The flow of work can be shown by them as in the flow diagram given below.

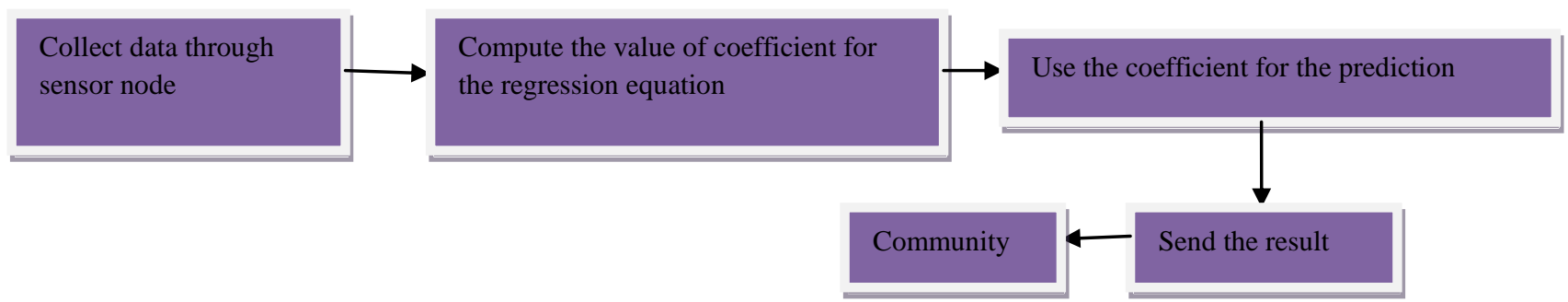

Fig.1 The process flow diagram of [1]

Here the author used multiple variable robust linear regression which is easy to understand and simple and cost effective in implementation, is speed efficient. It has low resources utilization and yet provides real time predictions with reliable accuracy, thus having features which are desirable in any real world algorithm.

The model is independent of the number of parameters, i.e. any kind and any number of parameters may be added or removed based on the on-site requirements. The rise in water level is represented by using a polynomial from which the exceeding of the flood line in the near future can be determined. In this paper a time multiplier function is used only to decide the time interval between two successive readings.

The central node is mentioned in this model but it is not taken into account. This model is only predicting the flooding situation and warning people about flood by ringing the alarm but it has no role in preventing the flooding situation. In this paper they have kept the efficient energy consumption part for future work.

\subsection{Honduras Flood Detection}

Basha et al. [2] presented a brief description about implementation of the sensor network in Honduras for an early detection of flood \& alert the community. They have analyzed on the significance on sensor networks in developing countries, sensor networks for flood detection and the available current operational systems for flood detection. This paper discussed about the flood detection problem of warning communities in impending disasters quickly becomes complex due to its multifaceted nature. They studied the flood detection problem in Honduras and proposed a solution.

Using wireless sensor network (WSN), they divided the solution into four tasks (event prediction, authority notification, community alert, and community evacuation) between CTSAR (name of NGO) and themselves.

They have conducted different experiments to validate the proposed solution. On the communication side, they verified the usability of the $144 \mathrm{MHz}$ radios. They tested it with the various ranges necessary for the system to ensure about the communication over those ranges.

To communicate at these ranges reliably, the radio antennas need line-of-sight high in the air, which requires antenna towers and limits the ability to test this portion of the system in the US. This paper says that wireless sensor network can be a perfect technology to be deployed for fighting with the flood in poor and developing country.
In this paper Bash et al have given a realistic idea on different situation of flood detection problem and employed different types of sensors in flood detection.

\subsection{Early Flood Warning System}

Basha et al. [3] describes a system architecture and deployment to meet the design requirements and to allow model-driven control for optimizing the prediction capability of the system. This architecture is used to explore the application of river flood prediction and it is describing the work on a centralized form of the prediction model, network implementation, component testing and infrastructure development in Honduras, deployment on a river in Massachusetts, and results of the field experiments. In this system a few number of nodes are deployed across river basin and a unique heterogeneous communication system is used for reading real-time sensed data, integrating self-monitoring for failure and adapting measurement schedules to capture events.

They proposed a model (Fig.2) and an efficient algorithm for flood prediction that uses data from the nodes of a spatially distributed sensor network. They have prepared this model in reference to Sacramento Soil Moisture Accounting (SACSMA) which is a very efficient model that can detect flood very easily but SAC-SMA is very expensive which could not be affordable for a developing country to be employed for flood detection. This approach is computationally simpler than conventional approaches to flood modeling and prediction, utilizing real-time data from multiple sensor nodes. This counts the advantage of this model over SAC-SMA.

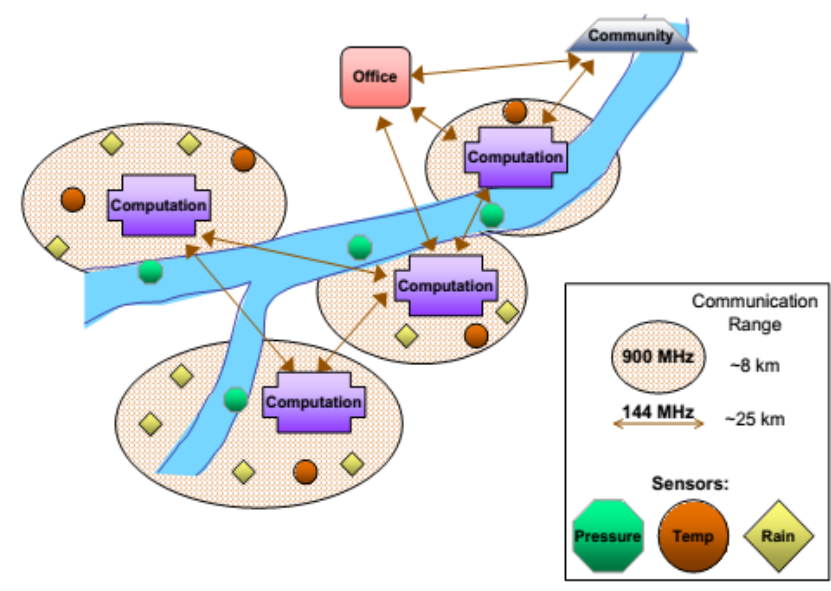

Fig.2 Idealized sensor network consisting of two communication tiers and four node types; communication ranges not to scale [3] 
They used the multiple linear regression models for flood forecasting which gives accurate and fast computation which adds to its advantage. The model is only based on rainfall driven flood so to predict flooding, a model requires knowing how much rain falls and what the soil's time dependent response to the rainfall will be. The model requires details of the soil composition, topography and land cover, along with meteorological conditions and hydro-meteorological quantities such as soil moisture. There are too many parameters which need to be sensed by sensors. This indicates that a number of different type of sensors are need to be employed which implies the expensiveness and complicated computation. This is the drawback of this model.

\subsection{Local and Remote Sensing}

Hughes et al. [4] described that damage due to flood is correlated to the warning time announced for a flood event. They prepared a hybrid of local and remote sensor network. For example, local computation can be used to provide timely warnings to local stakeholders and a combination of local and remote computation can inform adaptation of the sensor network to maintain optimal performance. The wireless sensor network for flood warning is capable of integrating with remote fixed-network grids for computationally-intensive flood modeling purposes and performs on-site flood modeling by organizing itself as a local grid [4].

The author proposed GridStix sensor platform which uses powerful embedded hardware, heterogeneous wireless networking technologies and next generation grid middleware to implement an adaptable WSN. It doubles as a lightweight grid, allowing nodes to not only ship data to remote fixed grids, but also to perform local grid computations.

They also describes about the use of local computation which can be used to

(i) inform system adaptation based on the environmental condition such as flood data and power monitoring

support diverse sensors i.e. by using an Image-based flow prediction. The flow rate and surface velocity can be calculated based upon a series of images taken by a digital camera. This technique is cheaper and easier to deploy than the ultrasound flow sensors. It is one of the advantages of this model.
Provides timely warnings to local stakeholders through on-site audio/visual warnings, a public web-site and SMS alerts.

For Image-based flow prediction a sequence of highresolution images is used. These are too large for off-site transmission to be feasible using GSM or GPRS technologies and therefore the method is impractical for this sensor network. It adds to the disadvantages part of the model.

The GridStix platform requires a strong embedded hardware. Each GridStix node is based on the Gumstix embedded computing platform which contains $400 \mathrm{MHz}$ Intel XScale PXA255 CPU, $64 \mathrm{Mb}$ of RAM and $16 \mathrm{Mb}$ of flash memory and a variety of hardware $\mathrm{I} / \mathrm{O}$ mechanisms, enabling connection to a variety of sensors. This indicates a complicated and expensive hardware structure which is counted as a disadvantage. About power consumption the whole structure runs at the expense of increased power consumption. This is another disadvantage.

\subsection{Alternative Emergency SMS Network}

Anthone et al. [5] described about an alternative network as a substitute to the usual communication links which are unavailable during major disaster. At the time of disaster, the communications for users inside the disaster zone would be almost to impossible because of no mobile network (Global System for Mobile Communications (GSM), Universal Mobile Telecommunications System (UMTS) or Long-Term Evolution(LTE)) still people need to communicate each other. So they proposed an alternative network for maintaining communications capabilities during major natural disasters and other emergency situations by a system that utilizes Short Message Service (SMS) of length upto 7bits over Wireless Mesh Sensor Networks (WMSNs). This is relatively simple and inexpensive.

To create this WMSN (as in fig.3) they propose a system using the water level sensors. The integration of the two noncellular wireless networks i.e. wireless sensor network and wireless mesh backbone is denoted as Wireless Mesh Sensor Networks (WMSNs). The topology was divided into two zone i.e. safe zone and disaster zone. The aim was to establish the interconnections between the WSN inside the disaster area and the mobile network infrastructure in the safe zone.

When a user initiates communication from the disaster zone, the user connects to the most nearby sensor inside a WSN using the ad-hoc application through WiFi directly to the sensor.

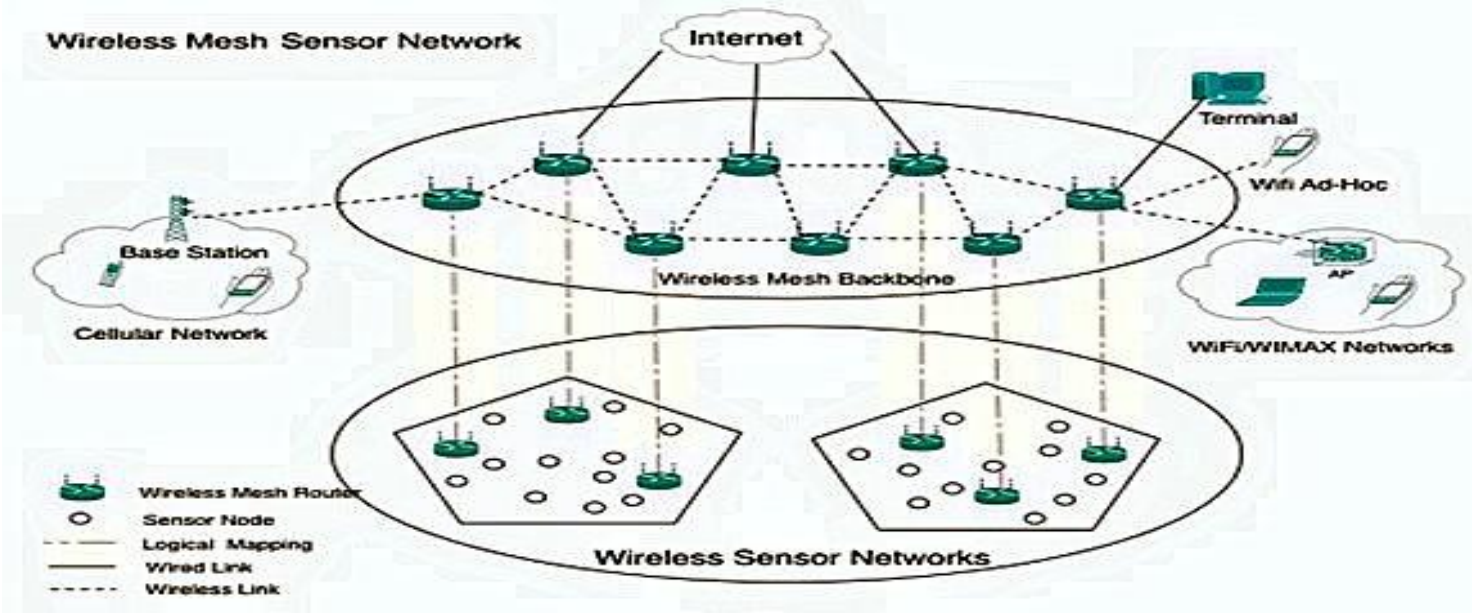

Fig.3 Network architecture of a Wireless Mesh Network with several access modes [5] 
The WSN becomes a medium in which devices communicate resiliently and independent of infrastructure (For example The internet or mobile network) in order to exchange data between networked objects and users in the disaster zone. The data is relayed from the WSNs in the disaster zone until it reaches the safe zone WSN and then the SMSG; then it interconnects with the mobile network infrastructure SMSC.

The SMSC relays the message to the user in the safe zone

One traffic analysis performance method was presented for WSN. In the deterministic network calculus model; network calculus is a min-plus system theory for deterministic queuing systems which is based on cluster topologies. The static wireless sensor network consisting of multiple sensor nodes, relaying nodes called cluster heads $(\mathrm{CH})$ and a sink node.

For the reliability of WSNs they used an operator-based deterministic performance analysis method to analyze integration of various duty cycle.

In this model they have not focused on the security and power consumption in sending a SMS through WMSN.

\subsection{Flood Monitoring and Warning System}

Sunkpho et al. [6] represented two main objectives of the developed system which serve a) as information channel for flooding between the involved authorities and experts to enhance their responsibilities and collaboration and b) as a web based information source for the public, responding to their need for information on water condition and flooding in Nakhon Si Thammarat, a southern province in Thailand.

The developed system (as in Fig.4) consists of three major components: sensor network, processing and transmitting modules and database and application server [6].

Sensor network measure water level and flow level by STAR flow sensor, and precipitation level through precipitation sensor by Fischer.

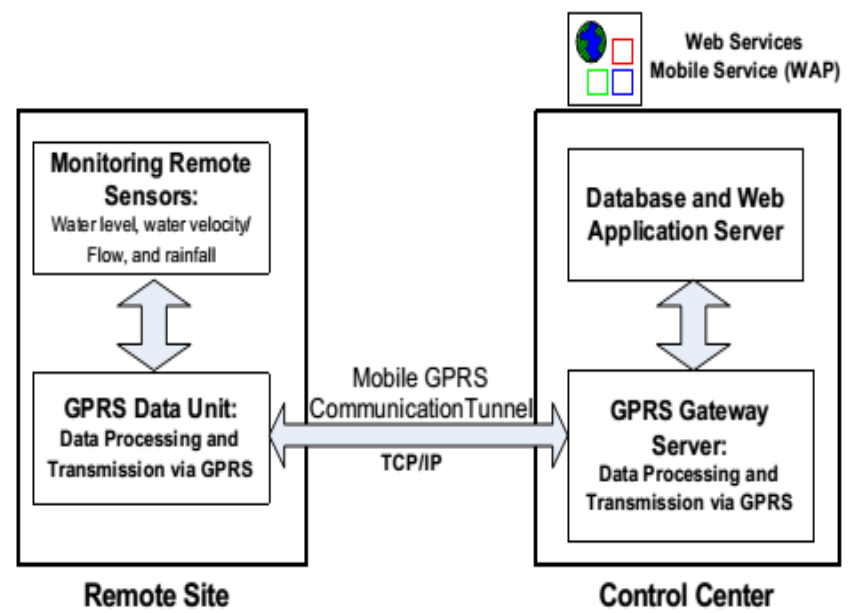

Fig.4 The highest-level system architecture [6]

Sensor network measure water level and flow level by STAR flow sensor, and precipitation level by precipitation sensor by Fischer.

Processing/transmission unit uses GPRS and virtualCOM (enables application server to communicate with the remote sensors connected to a GPRS data unit (GDU)).GPRS data unit (GDU) behaves like a cable which directly connected to the remote sensors to the application server using PHP and JAVA as the web application and MySQL as its relational database. Database/application servers process the measured data from sensor and make them accessible via web browser or WAP-enabled mobile phone.

Sunkpho et al have done a very noble work but they could have improved it by focusing on the reliability, adaptability, security and efficient power consumption in the real time flood monitoring and warning system.

In this approach there is no steps are taken regarding security purpose. This adds to the disadvantages of this developed system.

\subsection{Sensor Energy Autonomy}

Shebli et al [7] described a method to calculate the amount of energy consumption by sensor in a network, according to the data flow rate, the number of nodes and the distance between them. They proposed an efficient reduction in energy consumption within the linear sensor network. They studied an energy model for the energy consumed by the radio link of each node. They analyzed two topologies. The first one is with equidistant nodes hop and the other one with optimal spaces between the last nodes.

In Energy model they showed that the energy in sensor node is consumed in three main function i.e. acquisition, communication and data processing. They showed that the energy needed to transmit $1 \mathrm{~KB}$ over a $100 \mathrm{~m}$ distance is approximately equivalent to the energy necessary to carry out 3million instructions at a speed of 100million instructions per second (MIPS).They calculated number of packet relayed and the total energy cost for the system. They calculated optimal transmission range through efficient distribution of distance between the nodes for transmission of data. They showed that the total energy consumption in a linear sensor network may be reduced (by $15 \%$ to $38 \%$ ) using optimal spacing between nodes and suggested that the transmitter can dynamically adjust its transmission power.

They have not deal with the renewable energy sources which could be combinedly optimize the sensor battery life.

\subsection{Key Energy Consumption}

Halgamuge et al [8] presented a comprehensive energy model for wireless sensor networks. It is based on seven key energy consumption sources i.e. processing, communication, sensing, transient, logging, actuation and cluster formation. A comparative study on the lifetime of a sensor node of comprehensive model and other existing model is done in this paper. The model is used to evaluate energy consumption and node lifetime for a sensor network with fixed configuration. The validation is evaluated by simulation. The result shows that existing energy models over-estimate life expectancy of a sensor node by $30-58 \%$

Further following two observations are taken: a) the optimal number of clusters increases with the increase of free space fading energy

b) Finding the optimal number of clusters does not matters when free space fading energy is very low (less than 1670 $\mathrm{pJ} / \mathrm{bit} / \mathrm{m} 2$ ) but this contrasts for larger networks even if free space fading energy is low. Guidelines for efficient and reliable sensor network design as well as extension to a sensor network with rotating cluster heads are provided.

The comprehensive energy model and its application are applicable to a general sensor network with fixed cluster and 
single-hop transmission but there is no discussion about the multi hop transmission.

\subsection{GIS based Wireless Sensor Network}

Ahmad et al. [9] presented a comprehensive study of the flood analysis and prediction using Geographical Information system (GIS) i.e. they are using an Arc GIS simulation tool to identify pre and post disaster flood risk analysis and an Ad hoc wireless Sensor Network Architecture. A model is proposed for flood risk analysis and prediction for calculating the impact of flood damage in disaster hit regions.

In this research study various geographical information Systems which is designed for Flood disaster management are focused and necessary input parameters including soil moisture, air pressure, direction of wind, humidity and rain fall are analyzed. The proposed model is a very helpful model in predicting the upcoming disasters and too helps emergency and rescue authorities to take necessary actions for saving the life when critical condition arises.

The Proposed network Architecture for Flood risk Analysis consists of three sub types given as [9]

i) Wireless sensor network field,

ii) GIS based Emergency Response Data Base server and

iii) Remote sensing and satellite based infrastructure.

They have also proposed a mathematical model for flood analysis and prediction based on air temperature (T), humidity level $(\mathrm{H})$, air pressure $(\mathrm{P})$, wind speed $(\mathrm{W})$, snow melt $(\mathrm{S})$, rain fall $(\mathrm{R})$, run off process $(\mathrm{Ro})$, time scale $(\mathrm{t})$ as input parameters to find the flood prediction factor $(\mathrm{y})$. They took the high $(\mathrm{H})$ and low value $(\mathrm{L})$ of each input parameter in the mathematical model to predict flood by formulating mathematical equation.

The outcomes of the research study focuses on the integration of GIS with wireless sensor networks in flood analysis and prediction along with the deep literature review. A frame work model is proposed for flood risk analysis. The simulation for flood forecasting in different regions of Sind province using ARC GIS simulation tool is performed.

\subsection{Least Irrigation Control System}

Jadoon et al [10] presented a least cost framework of irrigation control system based on sensor network for efficient water management in Pakistan. As that there exists a direct relationship between irrigation and growth per yield of agriculture which implies the high demand of water in the country is directly proportional to scares water resources so an efficient management and maintenance of water resources and controlling the water wastage is required for agriculture. The proposed wireless sensor for irrigation control system will monitors four important environmental parameters like moisture, temperature, humidity, soil moisture, etc for the efficient management of water.

A prototype for irrigation control using sensor network is proposed. This model continuously monitors concentrated area through sensors by sensing the environmental parameters like temperature, ambient light, humidity, soil moisture etc in a interval of 20minute. Depending on the sensed value the area which is facing the shortage of water is detected. By adopting this approach the water is not wasted and water could be distributed to only those areas which need it. Several sensor nodes are deployed throughout the field. The aggregated data is processed and its packet is formed that contains Destination ID of the sink node aka target location of the packet. In this way water shortage that happens because of mismanagement can be controlled. The important characteristics of the irrigation model includes: :less time required for transformation of data, proper decision making, communicated primitives that provide addressing for sensor networks, authentication of nodes, next hope forwarding, and redundancy \& fault tolerance.

The proposed model was very easy to deploy, cost-effective and facilitates properly maintained irrigation system.

The major benefits of the proposed model are

1) Decreases labor for watering

2) Convenient

3) Complete and wherever required landscape coverage,

4) Technologically and easy control over irrigation timing for overnight or early-morning watering

5) Minimizes losses of the plants during deficiency.

\subsection{Pipe Net System}

Stoianov et al. [11] described a Pipe Net, a system based on wireless sensor networks which detect, localize and quantify bursts and leaks and other anomalies in water transmission pipelines such as blockages or malfunctioning control valves. The system monitors water quality in transmission, distribution water systems and the water level in sewer collectors.

The development process was divided into three stages (Fig.5). In first stage the development and field validation of a small-scale prototype deployment is done.

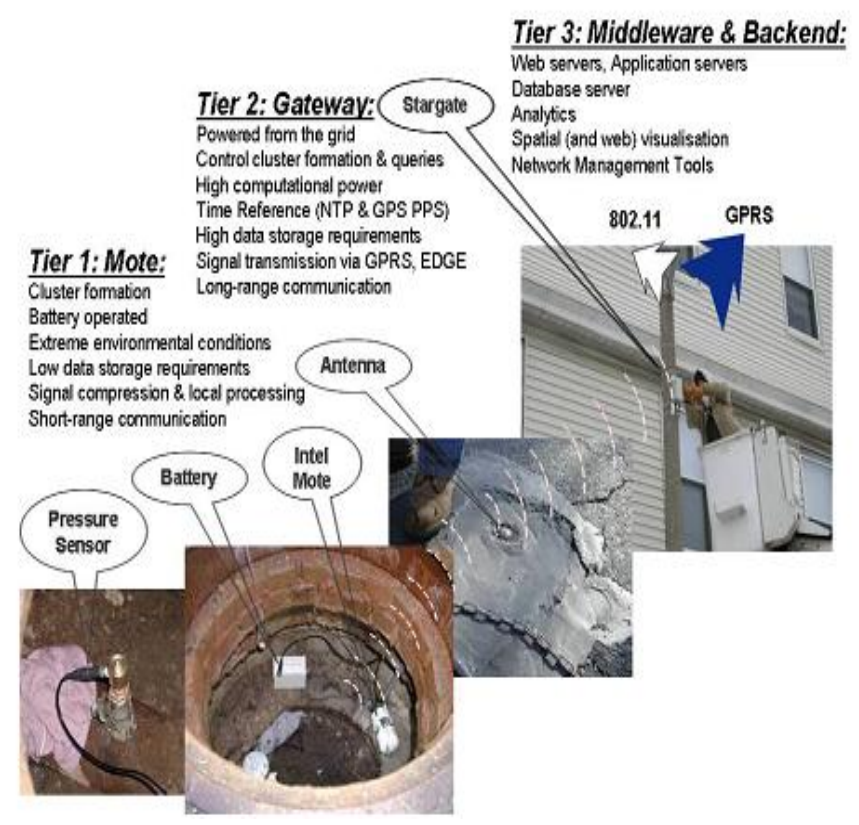

Fig.5 Overview of Pipe Net Deployment [11]

This consists of three clusters and each cluster had a single sensing node. In second stage, testing and validating some of the more advanced data acquisition, analysis techniques including time synchronized data collection and acoustic leak detection in a laboratory setting was done. In the third stage the results from the field and laboratory tests are being integrated, which will be a complete real-time monitoring solution. 
The Intel Mote2 platform was used that integrates many essential components to enable high performance, energy efficient data processing. Its XScale processor with dynamic voltage and frequency scaling capability that allows applications for a balanced performance and energy needs by regulating the speeds between $13 \mathrm{MHz}$ and $624 \mathrm{MHz}$. The Intel Mote 2 includes $32 \mathrm{MB}$ of SDRAM and Flash enabling the decoupling of data collection and communication and a richer peripheral support which will provide higher data acquisition rates and improve sensor integration. The new hardware platform along with the next generation in-network processing system will alter the way water utilities, monitor and maintain the pipeline infrastructure. This paper inspires us to make a better hardware platform and a wireless sensor network system for flood prediction and prevention.

\subsection{Brazil Flood Monitoring}

Degrossi et al. [12] proposed a model which is based on Open Geospatial Consortium's (OGC) Sensor Web Enablement (SWE) standards, that collects data to be shared in an interoperable and flexible manner.

A Spatial Data Infrastructure (SDI), geospatial software platforms which was used to manage the environmental risks.

There are two essential requirements for the development of an SDI in the context of flooding:

a) Interoperability: the need to integrate the heterogeneous data sources

b) Flexibility: the ability to adapt to the wide range of situations and specific needs of different scenarios.

A Service Oriented Architecture (SOA) was used to integrate data acquired from different types of sensors.

A new hardware platform is attempted with the aim of obtaining a good range of wireless communications between the nodes, with a good cost/benefit. Depending on this, the platform using Xbee motes is developed. This platform has the advantages such as lower energy consumption, a smaller physical size, and a greater range of communication between each node.

The OGC defines the Sensor Observation Service (SOS) as a service designed for the handling of data originating from sensors in an interoperable way. The development of the application is carried out by using the GeoExt API (Application Program Interface) as a base, i.e. a JavaScript open source library developed especially for creating geographic web application. The open layers library is used together with the GeoExt API.

The approach was deployed with two sensor nodes in the city of Sao Carlos/SP in order to monitor two rivers that cross the city, as can be seen in the prototype interface. The objective of this study was concerned in mapping out the river levels in the town of São Carlos with the aim of determining the existence of flooding in critical regions. This approach provides a real world application of wireless sensor networks and geospatial services to monitor the river water level in the town of São Carlos in Brazil, as well as to use the data supplied by the sensors aimed at detecting floods.

To overcome these above problems we have proposed a model for prediction and prevention of flood.

\subsection{Forest Fire Detection}

$\mathrm{Yu}$ et al. [13] presented a wireless sensor network for realtime forest fire detection method. The forecast forest fires cannot be detected by the satellites fire spreads uncontrollable. The wireless sensor network can detect and forecast forest fire more perfectly and accurately than the traditional satellitebased detection approach. This method is used to minimize the loss of forests, wild animals, and people in the forest fire.

A large number of sensor nodes are deployed in a forest to detect fire. Those are mainly cluster heads, sink, wind sensor and managing node. A few wind sensor nodes are manually connected to the sink via wired networks to detect wind speed. Sensor nodes collect measured data for example, environment temperature, relative humidity and smoke.

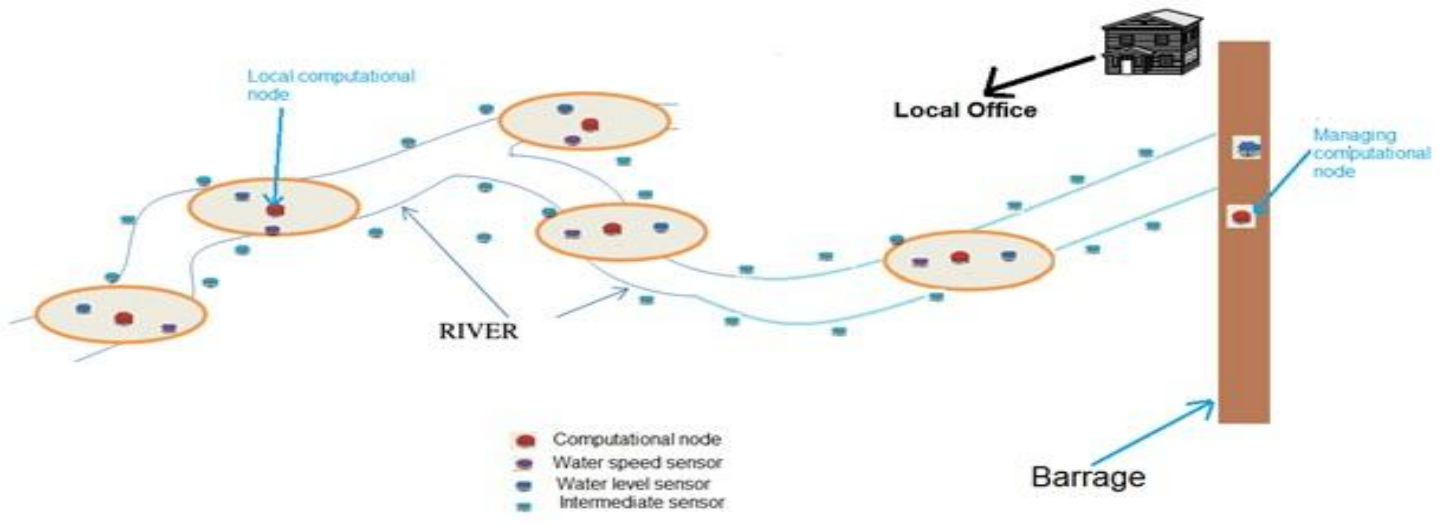

Fig.6 The proposed Model

These data along with location information are sent to their respective cluster heads. The neural network takes the measured data as input to produce weather index. This index measures the likelihood for the weather to cause a fire. Cluster headers will send weather indexes to a manager node via the sink. Finally the manager node concludes the forest fire danger rate based on received weather indexes and some other factors.

The manager node provides two types of information to users:
(1) Emergence report for abnormal event for example, smoke or extremely high temperature.

(2) Real-time forest fire danger rate for each cluster basing on the weather indexes and other forest fire factors.

The design part includes mainly three things that are clustering, data collection and data processing.

Clustering process uses a clustering algorithm for routing in wireless sensor network. Clustering routing helps the neural network method. 
In data collection, each node can generate three types of data packets:

(1) Regular Report (RR) - The sensing data is packaged into RR packet and send to the destination.

(2) Query Response (QR) - The QR packet is only sent to the sink after getting a query packet.

(3) Emergence Report (ER) - When any node detects any abnormal event, e.g. smoke is detected.

In data processing, the cluster head processes the packet according to the type of packets i.e. RR or $\mathrm{QR}$ or ER

They mainly divided the work of detecting the forest fire by wireless sensor network into two parts, In-networking Processing and forest fire detection. The simulation results show that our in-network processing approach is efficient to reduce communications between sensor nodes.

\section{PROPOSED APPROACH}

From the above survey on Wireless Sensor Network (WSN), we come to know about the cost effectiveness, faster and accurate computation \& easy deployment of sensor network in most of the real world phenomenon like traffic monitoring, billiard play ground etc. as well as in predicting and preventing natural calamities like flooding, earth quake etc.

This proposed model consists of three different types of nodes. Those are Sensing node, computational node and intermediate node. In this model (Fig.6) the data is collected dynamically through sensing nodes, data is transferred through computational node, water is managed to predict and prevent flood at barrage.

In this approach the sensing node reads the data like water level and water speed etc at a particular distance away from the barrage. Two types of computational nodes are used; first one is a local computational node which computes the volume and time of water flow to reach the barrage by using fluid flow principle [14]. Another computational node is a managing computational node which is deployed at the barrage that helps managing barrage water. The rate of flushing is computed at managing node and intimated to the local office. The intermediate node ensures the connectivity with the managing node. If the local computational node fails to transfer data to the managing computational node then the intermediate node helps in transferring the data. In this way the flood could be prevented due to sudden flushing of huge amount of water from barrage at a time.

\section{CONCLUSION}

The proposed model is an efficient model which helps in preventing flood due to sudden flush out of excess water at barrage at a time. In this model the water at barrage is flushed from the barrage in a controlled manner so that flood in the plain area will not occur. The advantages of this model are that we are using minimum number of parameter. We are also including the less battery energy consumption method.

This model is a cost effective model. Hence this can be deployed by developing and poor country to fight back with flood.

\section{REFERENCES}

[1] Victor Seal, Arnab Raha, Shovan Maity, Souvik Kr Mitra, Amitava Mukherjee and Mrinal Kanti Naskar. A simple flood forecasting scheme using wireless sensor networks. International Journal of Ad hoc, Sensor \&
Ubiquitous Computing (IJASUC) Vol.3, No.1, February 2012

[2] Basha, Elizabeth, and Daniela Rus. "Design of early warning flood detection systems for developing countries." Information and Communication Technologies and Development, 2007. ICTD 2007. International Conference on. IEEE, 2007.

[3] Basha, Elizabeth A., Sai Ravela, and Daniela Rus. "Model-based monitoring for early warning flood detection." Proceedings of the 6th ACM conference on Embedded network sensor systems. ACM, 5-7 November 2008.

[4] Danny Hughes, Phil Greenwood, Gordon Blair, Geoff Coulson, Florian Pappenberger, Paul Smith and Keith Beven. An Intelligent and Adaptable Grid-based Flood Monitoring and Warning System (DRAFT).UK eScience All Hands Meeting 5th, 2006

[5] Vinicio Anthone, Satoru Oishi. A wireless mesh sensor network framework for river flood detection which can be used as an emergency communications network in case of disaster. $11^{\text {th }}$ International Conference on Hydroinformatics HIC 2014

[6] Jirapon Sunkpho and Chaiwat Ootamakorn. Songklanakarin J. Sci. Real-time flood monitoring and warning system. Technol.33 (2), 227-235, Mar. - Apr. 2011

[7] F. Shebli, I. Dayoub and J.M. Rouvaen.Minimizing energy consumption within wireless sensors networks. Ubiquitous Computing and Communication Journal ,2007

[8] M.N.Halgamuge, M. Zukerman, and K. Ramamohanarao, H.L.vu. An estimation of sensor energy consumption. Progress in Electromagnetics Research B, Vol. 12, 259-295, 2009

[9] Naveed Ahmad, Mureed Hussain, Naveed Riaz, Fazli Subhani, Sajjad Haider, Khurram.S.Alamgir, Fahad Shinwari. Flood Prediction and Disaster Risk Analysis using GIS based Wireless Sensor Networks, A Review.Journal of Basic and Applied Scientific Research. ISSN 2090-4304, 2013

[10] Sultanullah Jadoon, Salman Faiz Solehria, Mubashir Qayum1. A Proposed Least Cost Framework of Irrigation Control System Based on Sensor Network for Efficient Water Management in Pakistan. International Journal of Basic \& Applied Sciences IJBAS-IJENS Vol: 11 No: 02. April, 2011

[11] Ivan Stoianov, Lama Nachman, Sam Madden. PIPENET: A Wireless Sensor Network for Pipeline Monitoring. IPSN'07, April 25-27, 2007.

[12] Lívia C. Degrossi, Guilherme G. do Amaral, Eduardo S. M. de Vasconcelos, João P. de Albuquerque, Jó Ueyama.Using Wireless Sensor Networks in the Sensor Web for Flood Monitoring in Brazil. Proceedings of the $10^{\text {th }}$ International ISCRAM Conference. May 2013

[13] Yu, Liyang, Neng Wang, and Xiaoqiao Meng. "Realtime forest fire detection with wireless sensor networks." Wireless Communications, Networking and Mobile Computing, 2005. Proceedings. 2005 International Conference on. Vol. 2. IEEE, 2005.

[14] A.K. Mohanty. Fluid Mechanics.Prentice-Hall of India Private Limited, Second edition 2004.

[15] K.C. Patra. Hydrology \& Water resources Engineering. 2 edition .Alpha Science Intl Ltd August 12, 2008 